\title{
Location cuing and response time distributions in visual attention
}

\author{
LAWRENCE R. GOTTLOB \\ University of Kentucky, Lexington, Kentucky
}

\begin{abstract}
The allocation of visual attention was investigated in two experiments. In Experiment $1(n=24)$, a peripheral cue was presented, and in Experiment $2(n=24)$, a central cue was used. In both experiments, cue validity was $90 \%$, and the task was four-choice target identification. Response time distributions were collected for valid trials over five cue-target stimulus onset asynchronies (SOAs), and ex-Gaussian parameters were extracted. In both experiments, only the mean of the Gaussian component decreased as a function of cue-target SOA, which implied a strict time axis translation of the distributions. The results were consistent with sequential sampling models featuring a variable delay in the onset of information uptake.
\end{abstract}

In location-cuing studies of visual attention, a particular location in the visual field is indicated (cued) by a marker, after which a target is presented. The most general finding from this type of study has been that target identification or detection is faster and more accurate at cued (valid) than at uncued (invalid) locations (Cheal \& Lyon, 1991; Jonides, 1981; Posner, 1980). A secondary finding has been that the time interval between cue and target onset (stimulus onset asynchrony, or SOA) affects both response time (RT) and accuracy. Specifically, as SOA increases, identification RT generally decreases at valid locations and increases or remains constant at invalid locations (Remington \& Pierce, 1984), whereas identification accuracy increases at valid locations and decreases or remains constant at invalid locations (Cheal \& Lyon, 1991; Gottlob \& Madden, 1998). The time signatures of these RT- and accuracy-SOA curves have been studied under a wide variety of stimulus and task demands (Cheal \& Lyon, 1991; Cheal, Lyon, \& Gottlob, 1994; Posner, 1980; for a review, see Wright \& Ward, 1998).

Much work on visual attention has involved an attempt to describe its movement or allocation, often employing metaphors, such as spotlight (Posner, Snyder, \& Davidson, 1980), zoom lens (Eriksen \& St. James, 1986), gradient (Cheal et al., 1994; LaBerge \& Brown, 1989), and stagelight (Sperling \& Weichselgartner, 1995). These metaphors for allocation, however, do not identify the mechanism by which attention improves processing of

This research was supported by National Institute on Aging Grant RO1 AG20860. The author is grateful for the helpful comments of John Cerella, Mario Fific, Robert Lorch, James T. Townsend, and Tom Zentall on a previous version of this article. Help on statistical issues was provided by Marie Gantz, Chris Saunders, and Kert Viele. Correspondence concerning this article should be addressed to L. R. Gottlob, Department of Psychology, University of Kentucky, Lexington, KY, 40506-0044 (e-mail: gottlob@uky.edu). the target. To address that issue in the context of target detection (simple RT), models have been formulated to distinguish between bias and sensitivity changes due to attention (Hawkins et al., 1990; Smith, 2000). On the other hand, when target identification, rather than detection, is measured, change in bias is no longer tenable as an explanation, and researchers have proposed mechanisms more directly involved with sensitivity. For instance, both Cheal (1997) and LaBerge (1994) proposed that attention works to increase the rate of information flow at the attended location. Other studies have suggested that attention works through such mechanisms as improved localization (Treisman \& Gelade, 1980) and exclusion of distractor information (Shiu \& Pashler, 1994).

In a few recent studies, attention mechanisms have been identified through the use of psychophysical functions relating target and distractor quality to accuracy. $\mathrm{Lu}$ and Dosher (2000) concluded that location cues improved identification accuracy because of perceptual effects, such as stimulus enhancement and external noise exclusion. Cameron, Tai, and Carrasco (2002) also found evidence for stimulus enhancement (which they called contrast gain) as the mechanism of attention. These researchers looked directly at improvements in accuracy due to effects on perceptual representations, but they did not examine directly the speeding-up of responses because of attention. This change in RT due to attention will be examined in the present study through the examination of RT distributions, which offer more information than do mean RTs.

RT distributions have been tied to underlying mechanisms through the use of sequential sampling models, such as the random walk and the diffusion models (Ratcliff, 1988; Ratcliff, Van Zandt, \& McKoon, 1999). Sequential sampling models for two-choice identification tasks are well established in the literature (for recent empirical work, see Ratcliff, 2002, Ratcliff, Thapar, \& McKoon, 2001, and Van Zandt, Colonius, \& Proctor, 2000; for analytic treatments, see Luce, 1986, and Townsend \& Ashby, 
1983). Random walk models treat information accumulation as occurring in discrete time; diffusion models are continuous generalizations. Both types of models have three major parameters: In the diffusion model, these are named drift rate, which is the rate of evidence accumulation, drift rate variance, and boundary, which is the amount of evidence required to emit a response. Each boundary in the standard two-choice model corresponds to a single choice (A or B); movement toward Boundary A implies movement away from Boundary B. A response is emitted when either boundary is crossed. In the two-choice diffusion model, information accumulation for A and B are perfectly (negatively) correlated. Ratcliff, who developed the two-dimensional diffusion model and fitted it to two-choice data, has added other factors, such as variability in starting state and contaminant RTs, in order to fit the model to data (Ratcliff, 2002; Ratcliff \& Tuerlinckx, 2002).

Generalizations of sequential sampling models to $n$ choices have been discussed by Luce (1986), Karpiuk, Lacouture, and Marley (1997), Shiffrin (1997), Nosofsky (1997), and Ratcliff and McKoon (1997). As Luce noted, $n$-choice models are potentially much more complex than two-choice models, especially if evidence accumulation is dependent among the choices (Shiffrin, 1997). However, as has been noted by several researchers (Marley \& Colonius, 1992; Van Zandt, 2002; Van Zandt et al., $2000)$, an $n$-choice random walk, with any degree of correlation among the choices, can be equivalently represented as a race across $n$ independent counters. (It should be noted, however, that there are restrictions on the types of models that can be mimicked by independent parallel processes; see Townsend, 1976.) According to this equivalence between dependent and independent parallel models, diffusion models and race models are not identifiably different, although if there is a reason to model particular dependencies between the choices (such as for fitting), they can serve to constrain a model. In that vein, Van Zandt et al. compared race and diffusion models for perceptual matching data and found that the fits were comparable for that particular data set. Many other fits of race models have been reported, including those of Karpiuk et al., Nosofsky, and Ratcliff and McKoon.

The task in the present paradigm is a four-choice identification task, which offers certain advantages over a twochoice identification task in the study of visual attention. These advantages include a lower chance rate of accuracy, which discourages guessing and increases the information value of locating the target. In accordance with the findings above regarding the equivalence of diffusion and race models, the four-choice identification can be modeled either by a four-boundary diffusion process with a complex (but underdetermined) correlation across responses or, alternatively, as a four-counter race model. The race model that most simply describes this task maps individual, independent counters to target responses (e.g., a Target $A$ counter produces only a Target $A$ response). This model contains two important features that preserve map- pings between single counters and responses: (1) The model does not converge counters onto responses, as opposed to some models that contain or processes (e.g., Response A could arise from Counter A or Counter B), and (2) the counters have context independence (Van Zandt, 2002), in that the marginal distributions for the single counters are identical to the single-counter distributions. Because the counters are nonconverging and context independent, RT distributions for correct trials can be assumed to be identical to the finishing time distributions of the single counters associated with each response. These finishing time distributions can be modeled by the one-dimensional (1-D) diffusion (Wiener) process (Luce, 1986; Ratcliff, 1993). The equation for this process (Cox \& Miller, 1965) expresses the RT probability density function (pdf), $g(t)$, as

$$
g(t)=\frac{L}{\sqrt{2 \pi d^{2} t^{3}}} \exp \left[\frac{-(L-r t)^{2}}{2 d^{2} t}\right],
$$

where $L$ is the boundary, $r$ is the drift rate, and $d$ is the drift rate variability. This distribution is called the Wald, or inverse Gaussian (Burbeck \& Luce, 1982; Luce, 1986; Ratcliff, 1993; Schwarz, 2001; Zabel \& Anderson, 1997) and fits empirical RT distributions well (Luce, 1986; Ratcliff, 1993).

In the interests of generality, it will be assumed that the four identification targets form an equivalence class, because the targets are $t$ shapes in different orientations, with minimal semantic content and equal visibility. This allows collapsing the counters for the four targets onto a single one-boundary diffusion process. This assumption, along with the direct mapping between component counters and responses, will allow us to simplify our model so as to draw general conclusions about the finishing time distributions of the 1-D diffusion process, which will be inferred from the shapes of RT distributions.

A useful distribution for describing RT distribution shape is the ex-Gaussian (Brown \& Heathcote, 2003; Hockley, 1984; Luce, 1986; Ratcliff, 1993; Ratcliff \& Murdock, 1976), which can closely approximate both the empirical RT distributions and the Wald. The ex-Gaussian is a distribution formed by the convolution of a Gaussian and an exponential distribution; the RT pdf, $f(t)$, is given by

$$
f(t)=\frac{e^{-[(t-\mu) / \tau]+\sigma^{2} /\left(2 \tau^{2}\right)}}{\tau \sqrt{2 \pi}} \int_{-\infty}^{\{[(t-\mu) / \sigma]-\sigma / \tau\}} e^{-y^{2} / 2} d y .
$$

It is determined by three parameters: $\mu$ and $\sigma$ (mean and variance of the Gaussian) and $\tau$ (exponential parameter). The mean of the ex-Gaussian is $\mu+\tau$, and the variance is $\tau^{2}+\sigma^{2}$. In describing RT distributions, variance is dominated by $\tau$, because $\tau$ is usually four times as large as $\sigma$ (Ratcliff, 1993).

In many cases, the ex-Gaussian fits both empirical RT distributions (Brown \& Heathcote, 2003) and diffusion model simulations (Ratcliff et al., 1999; Spieler, Balota, \& Faust, 2000) at least as well as any other distribution. The 
ex-Gaussian and the Wald are very close in appearance; often they can be distinguished only by examination of the hazard functions in the tails (Van Zandt, 2002). Furthermore, it will be shown in the Discussion section that changes in boundary and drift rate in the Wald map onto changes in the parameters of the ex-Gaussian.

Previous research in which the fitting of ex-Gaussians to RT distributions has been used has shown that the parameters seem to react in a lawful manner to experimental manipulations. For instance, Hockley (1984) found that for visual search, increasing set size produced increases in $\mu$ and $\sigma$, whereas for memory search, increasing set size produced increases in $\tau$ only. These differential effects were interpreted as reflecting a fixed search rate (visual search) versus a search rate that was affected by load (memory search). Similar differences, attributed to fixed- versus load-affected search rate, were found in judgment-of-recency and recognition tasks. In the same vein, Spieler et al. (2000) compared interference effects in the Stroop task (which involves nonspatial selectivity) with other tasks, such as global/local figures and flanker tasks (which involve spatial selectivity). Stroop interference in color naming produced increases in $\mu, \sigma$, and $\tau$, whereas interference in the four spatial tasks was manifested in $\mu$ and $\sigma$, but not in $\tau$. One interesting aspect of both Hockley's and Spieler et al.'s findings is that when there was an effect in $\mu$, there was always a corresponding effect in $\sigma$, indicating that when the Gaussian portion of the distribution was affected, it was always affected in both of its parameters. Many other researchers have used ex-Gaussian parameters to link experimental manipulations to RT distributions, including Ratcliff $(1988,1993)$ and Ratcliff and Murdock (1976).

In addition to the relationship between experimental manipulations and changes in ex-Gaussian parameters, there are mappings between parameter changes in the diffusion process (as represented in the present context by the Wald) and parameter changes in the ex-Gaussian. In the most general sense, a change in any single diffusion parameter will affect all three parameters of the ex-Gaussian. This relationship between the diffusion process and the ex-Gaussian will be explored more fully in the Discussion section of this article. In the present study, RT distributions from a location-cuing task were fitted with ex-Gaussians, and the attention effect, as reflected in the ex-Gaussian parameters, provided constraints on a diffusion model interpretation of the mechanisms involved in attention.

\section{EXPERIMENT 1}

Experiment 1 was designed to investigate attention in a location-cuing task by analyzing RT distributions for target identification. Previous research in which identification accuracy has been used has shown that accuracy for valid trials increases as a function of cue-target SOA and asymptotes at 100-150 msec for cues presented near the target (peripheral cues; Cheal \& Lyon, 1991; Gottlob
\& Madden, 1998). The implication has been that very short SOAs do not allow for sufficient attentional preparation by the time the target appears but that slightly longer SOAs allow for attentional processes to be fully engaged at target onset. The asymptotic behavior indicates that perceptual processes are affected maximally by attention at very short time scales.

Studies in which RT has been investigated have found that identification RT for valid trials also decreases over a similar time scale (Eriksen \& Hoffman, 1972; Posner, 1980), although asymptotic performance is difficult to confirm with RT, because of the possibility of generalized warning effects. The decrease in mean RT due to attention over a range of short cue-target SOAs should implicate (at least partially) the same perceptual processes that are involved with the increase in accuracy. If that is the case, the shapes of the RT distributions may also change as a function of SOA. For instance, if attentional engagement improves the perceptual representation of the target at longer SOAs, one might predict ex-Gaussian parameter changes that would be consistent with increases in drift rate in a diffusion process. In Experiment 1, RT distributions for five successive cue-target SOA values were compared in terms of shape, using ex-Gaussian decomposition.

Successive cue-target SOAs were chosen as a basis for comparison for several reasons. First, because the goal was to compare various levels of attentional preparation, three different potential baseline conditions were considered but rejected. (1) Invalid trials were considered as a baseline, as has been used previously in studies looking at RT distributions (e.g., Johnson \& Yantis, 1995). There are some potential pitfalls to this method, however, in that the responses to valid and invalid trials may be qualitatively different in some way. For instance, if probability matching were used by observers, valid and invalid trials would consist of different mixture distributions of discrete attentional states, such as spotlight versus spread of attention (Jonides, 1983; but see Johnson \& Yantis, 1995). This would make comparisons of RT distributions problematic. (2) Another possibility would be to use a neutral cue condition as a baseline and compare valid RTs with that, but there are methodological problems involved in comparing neutral with valid trials (Jonides \& Mack, 1984). For instance, a neutral cue consisting of four markers (one for each possible target location) may trigger a different type of attention allocation than does a cue that indicates a single target location with $90 \%$ probability. The only exception to the neutral/ valid distinction is when noninformative cues are used. (3) A third possible baseline that was rejected was that of a 0 -msec SOA trial (simultaneous cue and target). This comparison would also raise problems, because the perceptual representation of a simultaneous cue and target is very much different from the target presented alone. For instance, at a 0 -msec SOA for peripheral cues, there may be lateral masking between the cue and the target (Lyon, 1990), and with any type of cue, the total lumi- 
nance on 0 -msec SOA trials is higher than when the target is presented alone. Because of the potential problems with these three measures of baseline performance, it was decided that it would be best to compare RT distributions across different cue-target SOAs. In this manner, the trials would be comparable in every way, except for the time interval between the cue and the target.

Cue validity was fixed at $90 \%$; the $10 \%$ of the trials that were invalid allowed the comparison of invalid and valid RTs, to ensure that the observers were allocating in response to the cue. Another reason for using a $90 \%$ valid cue was that high probabilities (high cue predictabilities) have been found to induce stronger cuing effects (Gottlob, Cheal, \& Lyon, 1999), and if any probability-matching strategy were to be used (Johnson \& Yantis, 1995; Jonides, 1983), trials would consist mostly of a single type of response. This would minimize the mixing of RT distributions, which would raise problems for any analyses based on their shape.

\section{Method}

Observers. Twenty-four University of Kentucky students between 19 and 25 years of age participated. All the observers had a minimum of 12 years of education. Corrected near visual acuity was measured from a 4-ft distance. Acuity ranged between 4/4 and 4/8 for all the observers. The observers were paid $\$ 10$ per 1-h session.

Apparatus and Stimuli. The stimuli were presented on a Viewsonic PF775 color monitor, operating in $600 \times 800$ mode at $120 \mathrm{~Hz}$, controlled by a Pentium 4 computer. Responses were recorded on a standard IBM keyboard. An adjustable chinrest fixed the eye-toscreen distance at approximately $80 \mathrm{~cm}$, with the eye at center screen level. Eye movement was monitored with a video camera connected by cable to a monochrome monitor located at the experimenter's desk. During training sessions, the observers were instructed to suppress eye movements, which were observed to occur on fewer than $1 \%$ of the trials (which were not excluded or replaced).
The stimuli consisted of white characters on a black background. Luminance was fixed at $330 \mathrm{~cd} / \mathrm{m}^{2}$ for all the screen stimuli. Figure 1 contains a schematic of the events in a trial. The first display consisted of a fixation point, which was a $0.5^{\circ}$ dash in the center of the screen, presented for $1,000 \pm 50 \mathrm{msec}$. The $50-\mathrm{msec}$ variability was due to machine delay in setting up the visual stimuli for each trial, but within each trial, bitmaps loaded into video memory ensured accurate presentation time. A 33-msec cue appeared that was $1^{\circ}$ to the outside of one of the target locations. On $90 \%$ of the trials, the cue appeared adjacent to the location containing the target (valid trials), whereas on $10 \%$ of the trials, the cue appeared adjacent to the location diametrically opposite the target (invalid trials). Following a variable (blank screen) delay, the target $(100 \mathrm{msec})$, along with three distractors, appeared. SOAs (time between onsets of the cue and the target) were $33,66,83,100$, and $133 \mathrm{msec}$, presented randomly.

The target was a $\mathrm{T}$ subtending $2.2^{\circ}$ in one of four orientations (pointing right, left, up, or down), centered in one of four locations $2.6^{\circ}$ to the top, bottom, left, or right of the fixation point. The distractors consisted of $2.2^{\circ}$ plus $(+)$ signs at the other three locations. The distractors were included so that there would not be a single abrupt onset when the target appeared. Sole abrupt-onset targets may attract attention automatically (Yantis, 1993; but see Folk, Remington, \& Johnston, 1993) and would thus eliminate the need for a cue. Following the target and the distractors, a $2.3^{\circ}$ contour mask was presented, which consisted of an outline of the four superimposed targets, at all target and distractor locations. The observer's task was to identify the target by pressing one of four possible arrow keys on the numeric keypad, according to which way the tail of the target was pointing. The mask was terminated at observer response, and accuracy feedback was provided by a green + on correct trials and a red $\mathrm{X}$ on incorrect trials. Cue-target SOA, target location, and target orientation were presented in random order on each trial.

Procedure. The observers practiced for two sessions, and data were collected on the third. The observers were instructed that a short response time was of primary importance, a low error rate was secondary, and eye movements were to be suppressed. Eight blocks of 90 trials were presented, with observer-terminated breaks between blocks, to produce 648 valid trials and 72 invalid trials per observer.

Time

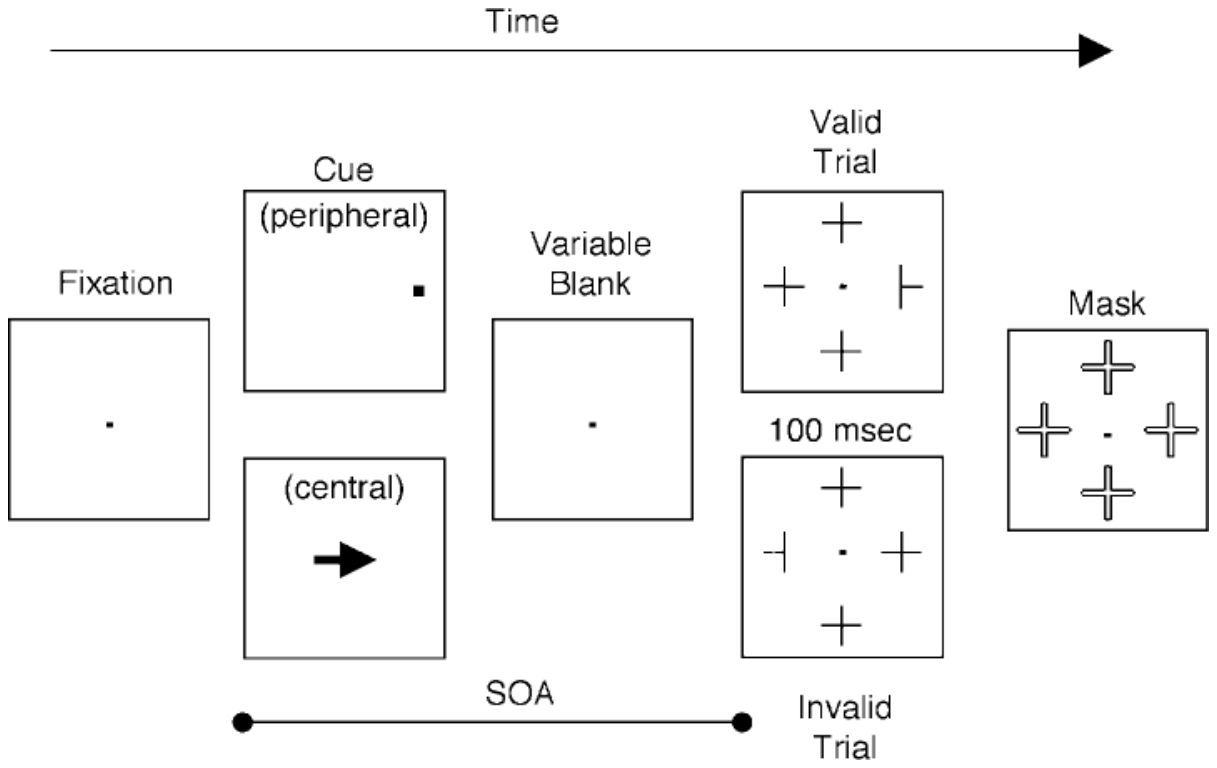

Figure 1. Order of events in a trial. Experiment 1 used peripheral cues; Experiment 2 used central cues. 


\section{Results and Discussion}

The results will be presented, followed by a brief discussion, for both Experiments 1 and 2. The General Discussion section will address the interpretations of the findings for both experiments.

Statistics. Because the observations (trials) were repeated within observers, the assumption of independence of observations was not met. In addition, cell means were not derived from equal numbers of observations; $90 \%$ of the trials were valid, and $10 \%$ were invalid. Therefore, the generalized linear mixed model, as implemented in PROC MIXED (Littell, Milliken, Stroup, \& Wolfinger, 1996), was employed. The variable of SOA was assumed to be randomly variable over observers, which yielded a repeated measures analysis over observers, equivalent to the standard repeated measures analysis of variance (ANOVA). The models implemented in PROC MIXED were evaluated using likelihood functions instead of sums of squares, as in an ANOVA. In PROC MIXED, generalized $F$ ratios are calculated using (restricted) maximum likelihood estimates of variance components and generalized least-squares estimates of treatment differences (Littell et al., 1996).

Mean accuracy as a function of cue-target SOA and cue validity is presented in Figure 2 (which also presents the results of the second experiment). There was no main effect of SOA, but there was a main effect of validity $[F(1,23)=1,461.53, p<.001]$, due to the higher accuracy for valid trials $(.97, S E=.18)$ than for invalid trials $(.68, S E=.17)$. There was a significant interaction of SOA and validity $[F(4,92)=2.99, p<.05]$. Preplanned simple effects analyses yielded no significant effect of SOA for invalid trials, but a significant effect of SOA for valid trials $[F(4,92)=2.60, p<.05]$. Post hoc paired comparisons among valid trial accuracies, using the Sidak correction (SAS Institute, 1989), yielded no pairwise differences at $p<.05$.

For RT analyses, fast responses (RT $<300 \mathrm{msec})$ and slow responses $(\mathrm{RT}>1,000 \mathrm{msec})$ were removed. This eliminated $1.6 \%$ of the observations for valid trials and $4.8 \%$ of the observations for invalid trials. The censoring did not materially change the results of the statistical analyses performed on the data. Mean correct RTs as a function of cue-target SOA and cue validity are presented in Figure 2. There were more total observations in the valid condition than in the invalid condition $(14,751$ vs. 1,085$)$, due to both the $9: 1$ valid:invalid trial ratio and the difference in accuracy across conditions. There was a significant main effect for SOA $[F(4,92)=7.19, p<.001]$ and a significant effect of validity $[F(1,23)=1,830.86$,



Figure 2. Mean correct response times (top) and accuracy (bottom) for valid and invalid trials. Experiment 1 used peripheral cues; Experiment 2 used central cues. Bars represent standard errors. 
$p<.001]$. The main effect of validity was due to a lower mean RT for valid trials ( $486.32 \mathrm{msec}, S E=13$ ) than for invalid trials $(612.49, S E=13)$. The validity $\times$ SOA interaction was not significant $[F(4,92)=1.73, p>.05]$.

In addition, preplanned simple effects analyses were run on RTs for valid and invalid trials separately. For invalid trials, there was no main effect of SOA $[F(4,92)=$ $0.39, p>.05]$; for valid trials, there was a main effect of SOA $[F(4,92)=25.26, p<.001]$, indicating that RTs decreased significantly as a function of SOA. Post hoc paired comparison tests of valid mean RT, using the Sidak correction, yielded significant differences for all pairs of observations, except for those between SOAs of 83 and $133 \mathrm{msec}, 83$ and $100 \mathrm{msec}$, and 100 and $133 \mathrm{msec}$. The maximum difference for the significant comparisons was $33 \mathrm{msec}$; the minimum difference was $9 \mathrm{msec}$.

As can be seen in Figure 2, valid trials produced higher accuracy and lower RTs than invalid trials did; this indicates that the observers were using the cue to allocate attention. In addition, RT decreased as a function of SOA for valid trials, but not for invalid trials, which indicates that the effects of attention are confined to valid locations for these SOAs. In previous research, it has been found that effects of peripheral cues are often maximal by an SOA of 100 msec (Cheal \& Lyon, 1991; Gottlob \& Madden, 1998; Jonides, 1981; Müller \& Rabbitt, 1989). It is impossible to establish asymptotic behavior with only a few data points; however, it appears in the present data that the benefit of the cue levels off by 100 or $133 \mathrm{msec}$.

Ex-Gaussian analysis. Because of errors, the mean number of observations for each RT distribution, per observer, was $123(S D=7.5)$. According to Brown and Heathcote (2003), the minimal number of observations required to produce good parameter estimates for exGaussians is about 40. Ex-Gaussians were fitted to the 120 individual RT distributions ( 24 observers $\times 5$ SOAs), using QMLE (Brown \& Heathcote, 2003; Heathcote, Brown, \& Mewhort, 2002) as the fitting procedure, and all fits converged to stable solutions. In order to evaluate the fits, Kolmogorov-Smirnov tests were performed on the data points versus the fitted values; the mean $p$ value was $.95(S D=.11)$, with a median of .99 . Of the 120 fits, $1 \mathrm{fit}$ had a $p$ value of less than .3, 1 was in the range of .4-.5, 6 were in the range of .7-.8, 5 were in the range of .8-.9, and 107 were between .9 and .999 . The KolmogorovSmirnov test is not sensitive to deviations in the tail, because it is based on the maximum deviation between data and predicted values. An alternative test, the Cramervon Mises (SAS Institute, 1989), is based on the integrated difference between data and predicted values and so has a little more sensitivity to differences in the tail. None of the Cramer-von Mises tests were significant at the .15 level. (It is generally accepted that a high $p$ value is not sufficient to accept a model; however, it may be used to infer that the model is sufficient to describe the data.) Means for the best-fitting values for $\mu$ and $\sigma$ (Gaussian mean and variance, respectively) and $\tau$ (exponential parameter), along with the standard errors for parameter estimates, are presented in Figure 3. Parameters $\sigma$ and $\tau$

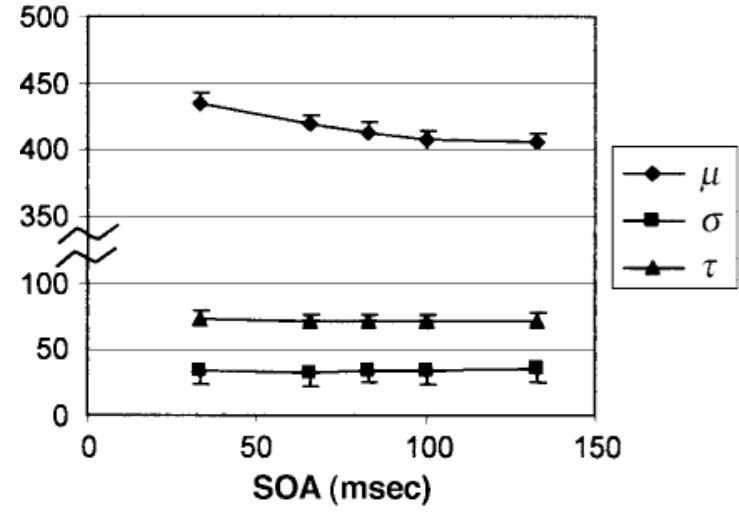

Figure 3. Means of best-fitting ex-Gaussian parameters for Experiment 1, valid trials, correct responses. Bars represent mean standard errors for parameter estimates, computed on individual fits.

do not change with SOA, but $\mu$ shows a consistent decrease with SOA. These parameter-SOA relationships were tested on the individual observer level for monotonic decreasing trends, using Kendall's $\tau$, which is an ordering coefficient based on paired comparisons (Gottlob \& Madden, 1999; Kendall, 1970). Kendall's $\tau$ ranges from -1 (strictly decreasing) to 1 (strictly increasing); random trends would have a mean score of 0 . Mean Kendall's $\tau$ values were $-.68, .05$, and -.05 for $\mu, \sigma$, and $\tau$, respectively, which indicates that the shapes of the individuals' RT distributions corresponded to those of the mean parameter estimates.

Figure 3 indicates that the two ex-Gaussian shape parameters $(\sigma$ and $\tau)$ were constant over SOA, whereas $\mu$, which determines the mean of the entire distribution, decreased as a function of SOA. This describes a translation of the distributions to the left as SOA increases, without any changes in shape. (One would also expect generalized warning effects to produce some sort of leftward translation as SOA increases, but these effects seem to be small or absent, because invalid RTs do not decline with SOA.) The leftward translation (change in $\mu$ ) is of a smaller magnitude than the change in SOA; increasing the SOA from 33 to $133 \mathrm{msec}$ only translated the distribution to the left by $30 \mathrm{msec}$. This yielded a mean $\mu-\mathrm{SOA}$ slope of .3; the maximum slope for any limb of the $\mu-\mathrm{SOA}$ curve was about 0.5 . Thus, it appears that RT changes produced by the response to a peripheral cue consist of similar RT distributions that vary only by translation along the time axis. The generality of this finding to another type of cue response will be examined in Experiment 2 . On the basis of both sets of results, it will be asserted in the General Discussion section that this pattern is consistent with a restricted class of sequential sampling mechanisms.

\section{EXPERIMENT 2}

In Experiment 1, a peripheral cue was used. Previous studies have shown that responses to peripheral cues are 
predominantly automatic (Yantis, 1993; but see Folk et al., 1993) and fast, as if attention is "pulled" to the target location. In contrast, responses to central cues are believed to require effort, because they must be interpreted first and then attention must be "pushed" to the target location. Whereas maximal responses to peripheral cues are often observed by a 100-msec cue-target SOA, in most cases the maximal response to central cues is observed only after $300 \mathrm{msec}$ (Cheal \& Lyon, 1991; Jonides, 1981; Müller \& Rabbitt, 1989). It has been suggested that responses to peripheral cues are mediated by a posterior visual cortical pathway, whereas central cue responses are mediated by an anterior visual cortical pathway (Posner, Walker, Friedrich, \& Rafal, 1984). The purpose of Experiment 2 was to test whether RT facilitation by a central cue would be manifested by a strict translation of the RT distribution to the left, as was found in Experiment 1 with a peripheral cue. This would be expected if, despite the differences in cue type and (presumably) the mechanism that processes the response to the cue, the manner in which attention speeds processing is the same.

\section{Method}

The observer demographics and apparatus were the same as those in Experiment 1. One observer had participated in Experiment 1; the rest were naive. The procedure was identical to that in Experiment 1 , except for the use of a central cue, instead of a peripheral cue, and the timing of the events on the computer screen (Figure 1). The cue consisted of a $1.7^{\circ}$ arrow with centroid at fixation, indicating one of four locations on the screen. The arrow was presented for $100 \mathrm{msec}$, followed by a 100 -msec target, with cue-target SOAs of $100,150,200,250$, or $350 \mathrm{msec}$. The targets and the masks were identical to those in Experiment 1.

\section{Results and Discussion}

Statistics. PROC MIXED analyses were performed as in Experiment 1. Mean accuracy as a function of cuetarget SOA and cue validity is presented in Figure 2 (which also presents the results from the first experiment). There was a main effect of validity $[F(1,23)=$ $1,287.51, p<.001]$, with valid trials $(M=.97, S E=$ .17) being higher in accuracy than invalid trials $(M=$ $.67, S E=.18)$. There was also a main effect of SOA $[F(4,92)=13.23, p<.001]$ and a significant interaction of SOA and validity $[F(4,92)=10.26, p<.05]$. Followup simple effects analyses showed no significant effect of SOA for invalid trials, but a significant effect of SOA for valid trials $[F(4,92)=37.55, p<.001]$. Post hoc paired comparisons $(p<.05)$ among valid trial accuracies, using the Sidak correction, yielded accuracy differences between an SOA of $100 \mathrm{msec}$ and all other SOAs and between an SOA of $150 \mathrm{msec}$ and all other SOAs. Maximum and minimum differences for the significant comparisons were .048 and .013, respectively.

As in Experiment 1, for RT (and ex-Gaussian) analyses, fast responses ( $\mathrm{RT}<300 \mathrm{msec}$ ) and slow responses $(\mathrm{RT}>1,000 \mathrm{msec})$ were removed. This eliminated $1.8 \%$ of the observations for valid trials and $7.6 \%$ of the observations for invalid trials. Mean correct RT as a function of cue-target SOA and cue validity is presented in Fig- ure 2. As in Experiment 1, there were more total observations in the valid condition than in the invalid condition $(14,531$ vs. 1,032$)$, due to both the $9: 1$ valid:invalid trial ratio and the difference in accuracy across conditions. In the PROC MIXED analysis, there was a significant main effect of SOA $[F(4,92)=6.96, p<.001]$ and a significant effect of validity $[F(1,23)=1,319.37, p<$ $.001]$. The main effect of validity was due to a lower mean RT for valid trials ( $526.74 \mathrm{msec}, S E=17)$ than for invalid trials $(643.52 \mathrm{msec}, S E=17)$. The validity $\times$ SOA interaction was significant $[F(4,92)=15.66, p<.001]$.

In addition, preplanned analyses were run on RTs for valid and invalid trials separately. For invalid trials, there was no main effect of SOA $[F(4,92)=0.13, p>.05]$; for valid trials, there was a main effect of SOA $[F(4,92)=$ $40.63, p<.001$ ], indicating that RT decreased significantly as a function of SOA. Post hoc paired comparison tests of valid mean RT, using the Sidak correction, yielded significant differences for all pairs of observations, with a maximum difference of $68 \mathrm{msec}$ and a minimum difference of $11 \mathrm{msec}$.

Just as in Experiment 1, valid trials produced higher accuracy and lower RTs than invalid trials did, which indicates that the observers were using the cue to allocate attention. Also, RTs decreased as a function of SOA for valid trials, but not for invalid trials, which indicates that the effects of attention were confined to valid locations. Thus, it appears that the central cues produced attention effects similar to those produced by the peripheral cue, but over a more extended time scale. In Experiment 1, the RT facilitation was effected by a strict translation of RT distributions along the time axis; therefore, an exGaussian analysis was performed on these data in order to compare results.

Ex-Gaussian analysis. As in Experiment 1, exGaussians were fitted to the individual observers' RT distributions for correct responses on valid trials, at each SOA, using QMLE. All 120 fits (24 observers $\times 5$ SOAs) converged successfully; mean parameter values are shown

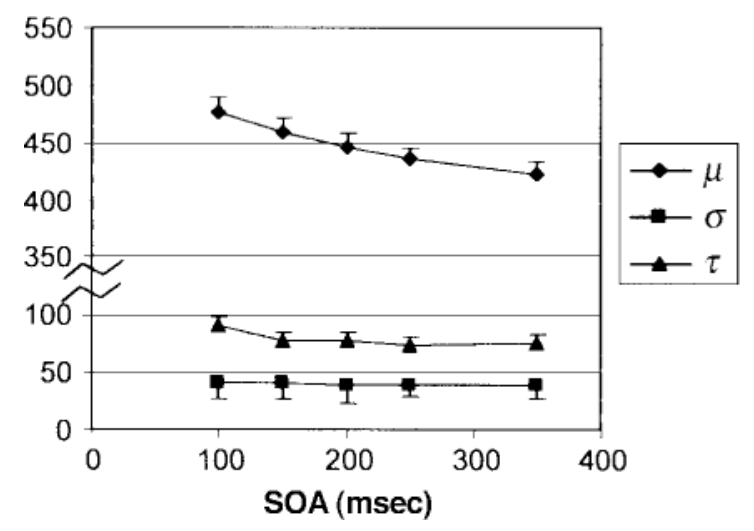

Figure 4. Means of best-fitting ex-Gaussian parameters for Experiment 2, valid trials, correct responses. Bars represent mean standard errors for parameter estimates, computed on individual fits. 
in Figure 4. Kolmogorov-Smirnov tests yielded a mean $p$ value of $.97(S D=.06)$, with a median of .998 . Of the 120 fits, 1 fit had a $p$ value of less than $.7,3$ were in the range of .7-.8, 7 were in the range of .8-.9, and 109 were between .9 and .999 . Cramer-von Mises tests were also performed; none were significant at the .15 level. As in Experiment 1, parameters $\sigma$ and $\tau$ do not change with SOA (except for a 12-msec change in $\tau$ between SOAs of 100 and $150 \mathrm{msec}$ ), but $\mu$ shows a consistent decrease with SOA. Applying Kendall's $\tau$ test for monotonicity yielded values of $-.79,-.04$, and -.29 for $\mu, \sigma$ and $\tau$, respectively, again indicating that the distributions on the individual level were consistent with the means.

Similar to the findings in Experiment 1, increasing the SOA from 100 to $350 \mathrm{msec}$ had the effect of translating the RT distribution to the left by $55 \mathrm{msec}$. The maximum $\mu-$ SOA slope for any individual limb was about 0.37 , as $\mu$ decreased from 477 to 458 between SOAs of 100 and $150 \mathrm{msec}$. Because $\tau$ also decreased by $12 \mathrm{msec}$ over the interval $(\mu+\tau=$ mean RT), the RT-SOA slope between those two SOA values was 0.62 .

\section{GENERAL DISCUSSION}

The results from both Experiments 1 and 2 are consistent with previous studies of location cuing and visual attention: Mean RT was lower for valid trials than for invalid trials, and valid RT declined as a function of cue-target SOA. The SOA values that were chosen $(33-133 \mathrm{msec}$ for peripheral cues and $100-350 \mathrm{msec}$ for central cues) corresponded to the findings in previous research that showed that identification accuracy reaches asymptotic values during this time interval (Cheal \& Lyon, 1991), meaning that perceptual processes subject to attention are not affected at later SOAs. Therefore, the processes that bring about a decrease in valid RT during this time interval probably have a large perceptual component. In addition, generalized warning effects may have contributed to the decrease in valid RTs, but if they existed, they were not apparent in the invalid mean RTs.

The present experiments expanded mean RT to a complete RT distribution, which revealed a constancy in distribution shape as a function of cue-target SOA. To summarize the RT results, in Experiment 1 (peripheral cue), the RT distribution shifted to the left ( $\mu$ decreased) by $30 \mathrm{msec}$ as SOA increased from 33 to $133 \mathrm{msec}$, and in Experiment 2 (central cue), the $\mu$ shift was $55 \mathrm{msec}$ as SOA increased from 100 to $350 \mathrm{msec}$. Both types of cues produced a leftward shift of the RT distribution without any changes in shape; there was a decrease in $\mu$, but no changes in $\sigma$ or $\tau$, with SOA (except for a change in $\tau$ over the first two SOAs in Experiment 2). These results may be contrasted with those of Hockley (1984) and Spieler et al. (2000), who always found changes in $\sigma$ when the value of $\mu$ varied.

It is possible that probing a larger range of SOAs might produce evidence for RT distribution shape changes on valid trials. Those changes, however, would be difficult to differentiate from generalized warning effects, because as was stated above, perceptual changes due to attention seem to be asymptotic by $100-150 \mathrm{msec}$ for peripheral cues and 300-350 msec for central cues (Cheal \& Lyon, 1991; Cheal et al., 1994). Also, longer SOAs might permit eye movements to occur, which would change the character of late-SOA responses.

The shape constancy of the present experiments, when put into the context of a diffusion model, may reveal the mechanisms at work in visual attention that serve to decrease identification RT. Because the diffusion model has been the preferred model to account for identification RT (e.g., Ratcliff, 2002), it was important to determine whether it could easily account for the present results. As was stated previously, the pdf of the 1-D diffusion process is described by the Wald distribution (Equation 1), which has three parameters: $L$ (boundary), $r$ (drift rate), and $d$ (drift rate variability). In order to explore the behavior of the Wald with respect to its parameters, many representative Wald pdfs, over a large variety of parameter values, were constructed over the interval $x=0-1 \mathrm{sec}$. Then, for each distribution, 1,000 simulated observations were sampled and fitted to ex-Gaussians. Fits were excellent between the sampled Wald values and the exGaussians (mean $r=.99$ ). It was found that varying a single Wald parameter $(L, r$, or $d)$ in order to reduce mean finishing time (RT) produced characteristic ex-Gaussian parameter changes. Decreasing the boundary produced decreases in $\mu$ and $\sigma$, with $\tau$ approximately constant. Increasing the drift rate (the most likely candidate for an attention-related parameter) produced decreases in $\mu, \tau$, and $\sigma$. Increasing drift rate variability produced a decrease in $\mu$, an increase in $\tau$, and an increase in $\sigma$. According to the simulations, it is not possible to create, by varying a single parameter, a family of identically shaped Wald distributions that differ only in location along the $x$-axis (i.e., where $\mu$, and no other parameter, in the fitted ex-Gaussian is changed). This result can be confirmed by comparing Equations 1 and 2. For Equation 2 (ex-Gaussian), $\mu$ is additive with respect to $t$, so the effects of changing $\mu$ and $t$ are equivalent. On the other hand, for Equation 1 (Wald), no parameter has a simple additive relationship with $t$.

The results of the above simulations and inspection of the Wald and ex-Gaussian equations run parallel to previous inferences drawn about the diffusion model and ex-Gaussian parameters. Zabel and Anderson (1997) plotted Wald densities and observed that, with changes in drift rate and drift rate variability, there were always corresponding changes in distribution shape. Ratcliff (1993) noted that changes in the Wald drift rate corresponded to changes in both $\mu$ and $\tau$ in the ex-Gaussian fits. Similarly, Spieler et al. (2000) simulated random walks in which single parameters (drift rate or boundary) were manipulated, after which they fitted ex-Gaussians. When boundary was increased, there were monotonic increases in $\mu, \tau$, and $\sigma$, although the increase in $\mu$ was about five times the increases in $\tau$ and $\sigma$. Spieler et al. 
also found that when they increased drift rate, there were monotonic decreases in $\mu, \tau$, and $\sigma$, although the decrease in $\tau$ was highly nonlinear over the range of drift rates they tested. These three studies confirm the above results that single parameter changes in the Wald would not produce simple $x$-axis translations of RT distributions. Of course, it is possible that coordinated parameter changes could effect a strict translation in the RT distributions, but these would have to take effect over all SOAs and both experiments. Therefore, the shape invariance of the RT distributions suggests that the diffusion process cannot model the present data by varying drift rate, drift rate variability, or boundary singly. This result was not anticipated, because drift rate is the most reasonable candidate for a diffusion model parameter that corresponds to differences in attention or task difficulty.

One way for a diffusion model to produce a set of translated RT distributions is by adding a parameter that corresponds to a variable delay in the onset of information uptake (drift). The delay would be longest at short cue-target SOAs. In some ways, this delay corresponds to hypothesized mechanisms in visual attention. For instance, Sperling and Weichselgartner (1995) suggested that a threshold amount of target information must be collected before identification processes can start, and it is reasonable that this delay would be greatest at short SOAs. In order for this delay to produce strictly translated RT distributions, it is necessary that the amount of noise accumulated during the delay remain constant. However, the few sequential sampling models that include a variable delay, which has been termed premature sampling (Laming, 1968; Rouder, 1996) or, equivalently, starting point variability (Ratcliff \& Rouder, 1998; Ratcliff et al., 1999), also posit that the system drifts, with mean drift rate equal to zero during the delay. This drift would add noise to the process in proportion to the amount of time spent in drift (or the starting point variance), which would show up in differences of $\sigma$ and $\tau$ in the ex-Gaussians.

In order to accommodate the shape constancy in the RT distributions that were found in the present experiments, a diffusion model would need to hold the total accumulated noise to a constant amount. This could be accomplished with a variable initial dwell period in which the integrated noise would be held to 0 or some other constant; that is, the system would be "on hold" for a variable period of time before information accumulation would start, perhaps waiting for target localization or information of a minimum "packet size." This constant-noise delay, although allowing the diffusion model to fit the present data, may seem biologically implausible and ad hoc.

In summary, the same results and implications were obtained for both peripheral (Experiment 1) and central (Experiment 2) cues: Decreases in mean identification RTs as a function of cue-target SOA were accomplished by an $x$-axis translation of the RT distributions. This similarity across experiments suggests that the two types of cues invoke common mechanisms affecting RT in target identification, but this mechanism does not appear to correspond with simple changes in sensitivity (drift rate) in a diffusion model. In order to accommodate the translated RT distributions, a model based on a diffusion process may be required to include constant-noise (with the degenerate case being zero-noise) variable time delays in the uptake of information. This may seem implausible biologically, but adjusting diffusion model parameters of drift rate, drift rate variability, or boundary (unless precisely coordinated) would have corresponded to changes in the shape of RT distributions as a function of SOA.

\section{REFERENCES}

Brown, S., \& Heathcote, A. (2003). QMLE: Fast, robust, and efficient estimation of distribution functions based on quantiles. Behavior Research Methods, Instruments, \& Computers, 35, 485-492.

BURBECK, S. L., \& LUCE, R. D. (1982). Evidence from auditory simple reaction times for both change and level detectors. Perception \& Psychophysics, 32, 117-133.

CAMERon, E. L., TAI, J. C., \& CARrasco, M. (2002). Covert attention affects the psychometric function of contrast sensitivity. Vision Research, 42, 949-967.

CHEAL, M. (1997). Understanding diverse effects of visual attention with the VAP-filters metaphor. Consciousness \& Cognition, 6 , 348362.

Cheal, M., \& Lyon, D. R. (1991). Central and peripheral precuing of forced-choice discrimination. Quarterly Journal of Experimental Psychology, 43A, 859-880.

Cheal, M., Lyon, D. R., \& Gottlob, L. R. (1994). A framework for understanding the allocation of attention in location-precued discrimination. Quarterly Journal of Experimental Psychology, 47A, 699-739.

Cox, D. R., \& Miller, H. D. (1965). The theory of stochastic processes. New York: Wiley.

Eriksen, C. W., \& Hoffman, J. E. (1972). Temporal and spatial characteristics of selective encoding from visual displays. Perception \& Psychophysics, 12, 201-204.

ERIKSEN, C. W., \& ST. JAMES, J. D. (1986). Visual attention within and around the field of focal attention: A zoom lens model. Perception \& Psychophysics, 40, 225-240.

FoLK, C. L., Remington, R. W., \& Johnston, J. C. (1993). Contingent attentional capture: A reply to Yantis (1993). Journal of Experimental Psychology: Human Perception \& Performance, 19, 682-685.

Gottlob, L. R., Cheal, M., \& Lyon, D. R. (1999). Time course of location-cuing effects with a probability manipulation. Journal of General Psychology, 126, 261-270.

GotTLOB, L. R., \& MADDEN, D. J. (1998). Time course of allocation of visual attention after equating for sensory differences: An age-related perspective. Psychology \& Aging, 13, 138-149.

GotTlOB, L. R., \& MADDEN, D. J. (1999). Age similarities in the inertial properties of attention. Perception \& Psychophysics, 61, 740-755.

Hawkins, H. L., Hillyard, S. A., Luck, S. J., Mouloua, M., DownING, C. J., \& WoODWARD, D. P. (1990). Visual attention modulates signal detectability. Journal of Experimental Psychology: Human Perception \& Performance, 16, 802-811.

Heathcote, A., Brown, S., \& Mewhort, D. J. K. (2002). Quantile maximum likelihood estimation of response time distributions. Psychonomic Bulletin \& Review, 9, 394-401.

HoCKLEY, W. E. (1984). Analysis of response time distributions in the study of cognitive processes. Journal of Experimental Psychology: Learning, Memory, \& Cognition, 10, 598-615.

JoHnSON, D. N., \& YANTIS, S. (1995). Allocating visual attention: Tests of a two-process model. Journal of Experimental Psychology: Human Perception \& Performance, 21, 1376-1390.

JoNIDES, J. (1981). Voluntary versus automatic control over the mind's eye's movement. In J. [B.] Long \& A. [D.] Baddeley (Eds.), Attention and performance IX (pp. 189-203). Hillsdale, NJ: Erlbaum. 
JONIDES, J. (1983). Further toward a model of the mind's eye's movement. Bulletin of the Psychonomic Society, 21, 247-250.

JONIDES, J., \& MACK, R. (1984). On the cost and benefit of cost and benefit. Psychological Bulletin, 96, 29-44.

Karpiuk, P., JR., Lacouture, Y., \& Marley, A. A. J. (1997). A limited capacity, wave equality, random walk model of absolute identification. In A. A. J. Marley (Ed.), Choice, decision, and measurement: Essays in honor of R. Duncan Luce (pp. 279-299). Mahwah, NJ: Erlbaum.

Kendall, M. G. (1970). Rank correlation methods (4th ed.). London: Griffin.

LABERGE, D. (1994). Quantitative models of attention and response processes in shape identification tasks. Journal of Mathematical Psychology, 38, 198-243.

LABERGE, D., \& BROWN, V. (1989). Theory of attentional operations in shape identification. Psychological Review, 96, 101-124.

LAMING, D. R. J. (1968). Information theory of choice reaction times. London: Academic Press.

Littell, R. C., Milliken, G. A., Stroup, W. W., \& Wolfinger, R. D. (1996). SAS system for mixed models. Cary, NC: SAS Institute.

Lu, Z.-L., \& Dosher, B. A. (2000). Spatial attention: Different mechanisms for central and peripheral temporal precues? Journal of Experimental Psychology: Human Perception \& Performance, 26, 1534-1548.

LuCE, R. D. (1986). Response times: Their role in inferring elementary mental organization. New York: Oxford University Press.

LYON, D. R. (1990). Large and rapid improvement in form discrimination accuracy following a location precue. Acta Psychologica, 73, 6972 .

Marley, A. A. J., \& Colonius, H. (1992). The "horse race" random utility model for choice probabilities and reaction times, and its competing risks interpretation. Journal of Mathematical Psychology, $\underline{\mathbf{3 6}}_{2}$ $1-20$.

MülleR, H. J., \& Rabbitt, P. M. A. (1989). Reflexive and voluntary orienting of visual attention: Time course of activation and resistance to interruption. Journal of Experimental Psychology: Human Perception \& Performance, 15, 315-330.

NosOFSKY, R. M. (1997). An exemplar-based random-walk model of speeded categorization and absolute judgment. In A. A. J. Marley (Ed.), Choice, decision, and measurement: Essays in honor of R. Duncan Luce (pp. 347-365). Mahwah, NJ: Erlbaum.

Posner, M. I. (1980). Orienting of attention. Quarterly Journal of Experimental Psychology, 32, 3-25.

PoSNER, M. I., SNYDER, R. R., \& DAVIDSON, D. J. (1980). Attention and the detection of signals. Journal of Experimental Psychology: General, 109, 160-174.

Posner, M. I., WalKer, J. A., Friedrich, F. J., \& Rafal, R. D. (1984). Effects of parietal injury on covert orienting of attention. Journal of Neuroscience, 4, 1863-1874.

RATCLIFF, R. (19 8). Continuous versus discrete information processing: Modeling the accumulation of partial information. Psychological Review, 95, 238-255.

RATCLIFF, R. (1993). Methods for dealing with reaction time outliers. Psychological Bulletin, 114, 510-532.

RATCLIFF, R. (2002). A diffusion model account of response time and accuracy in a brightness discrimination task: Fitting real data and failing to fit fake but plausible data. Psychonomic Bulletin \& Review, 9, 278-291.

RATCLIFF, R., \& McKoon, G. (1997). A countermodel for implicit priming in perceptual word identification. Psychological Review, 104, 319-343.

RATCLIFF, R., \& Murdock, B. B., JR. (1976). Retrieval processes in recognition memory. Psychological Review, 83, 190-214.

RATCLIFF, R., \& Rouder, J. N. (1998). Modeling response times for two-choice decisions. Psychological Science, 9, 347-356.
Ratcliff, R., Thapar, A., \& McKoon, G. (2001). The effects of aging on reaction time in a signal detection task. Psychology \& Aging, 16, 323-341.

RATCLIFF, R., \& TUERLINCKX, F. (2002). Estimating parameters of the diffusion model: Approaches to dealing with contaminant reaction times and parameter variability. Psychonomic Bulletin \& Review, 9 , 438-481

RATClifF, R., VAN ZANDT, T., \& McKoon, G. (1999). Connectionist and diffusion models of reaction time. Psychological Review, $\mathbf{1 0 6}_{2}$ 261-300.

REMINGTON, R., \& Pierce, L. (1984). Moving attention: Evidence for time-invariant shifts of visual selective attention. Perception \& Psychophysics, 35, 393-399.

RoUDER, J. N. (1996). Premature sampling in random walks. Journal of Mathematical Psychology, 40, 287-296.

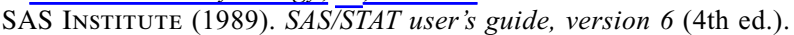
Cary, NC: Author.

Schwarz, W. (2001). The ex-Wald distribution as a descriptive model of response times. Behavior Research Methods, Instruments, \& Computers, 33, 457-469.

ShIFFrIN, R. M. (1997). A network model for multiple choice: Accuracy and response times. In A. A. J. Marley (Ed.), Choice, decision, and measurement: Essays in honor of R. Duncan Luce (pp. 329-346). Mahwah, NJ: Erlbaum.

ShiU, L., \& PAshler, H. (1994). Negligible effect of spatial precuing on identification of single digits. Journal of Experimental Psychology: Human Perception \& Performance, 20, 1037-1054.

SMITH, P. L. (2000). Attention and luminance detection: Effects of cues, masks, and pedestals. Journal of Experimental Psychology: Human Perception \& Performance, 26, 1401-1420.

SPERLING, G., \& WeICHSELGARTNER, E. (1995). Episodic theory of the dynamics of spatial attention. Psychological Review, 102, 503-532.

Spieler, D. H., Balota, D. A., \& Faust, M. E. (2000). Levels of selective attention revealed through analysis of response time distributions. Journal of Experimental Psychology: Human Perception \& Performance, 26, 506-526.

TowNSEND, J. T. (1976). Serial and within-stage independent parallel model equivalence on the minimum completion time. Journal of Mathematical Psychology, 14, 219-238.

Townsend, J. T., \& Ashby, F. G. (1983). Stochastic modeling of elementary psychological processes. Cambridge: Cambridge University Press.

Treisman, A., \& Gelade, G. (1980). A feature integration theory of attention. Cognitive Psychology, 12, 97-136.

VAN ZANDT, T. (2002). Analysis of response time distributions. In H. Pashler (Series Ed.) \& J. T. Wixted (Vol. Ed.), Stevens' Handbook of experimental psychology: Vol. 4. Methodology in experimental psychology (3rd ed., pp. 461-516). New York: Wiley.

VAn Zandt, T., Colonius, H., \& Proctor, R. W. (2000). A comparison of two response time models applied to perceptual matching. Psychonomic Bulletin \& Review, 7, 208-256.

WRIGHT, R. D., \& WARD, L. M. (1998). The control of visual attention. In R. D. Wright (Ed.), Visual attention (pp. 132-186). New York: Oxford University Press.

YANTIS, S. (1993). Stimulus-driven attentional capture and attentional control settings. Journal of Experimental Psychology: Human Perception \& Performance, 19, 676-681

ZABEL, R. W., \& ANDERSON, J. J. (1997). A model of the travel time of the migrating juvenile salmon, with an application to Snake River spring chinook. North American Journal of Fisheries Management, 17, 93-100.

(Manuscript received May 28, 2003;

revision accepted for publication January 12, 2004.) 